\title{
Clinical evaluation of bulk-fill composite resin and layered composite resin restoration in class I cavity of permanent molar teeth
}

\author{
Mohammad Fida Hasan Talukder, Mozammal Hossain and Mohammad Ali Asgor Moral
}

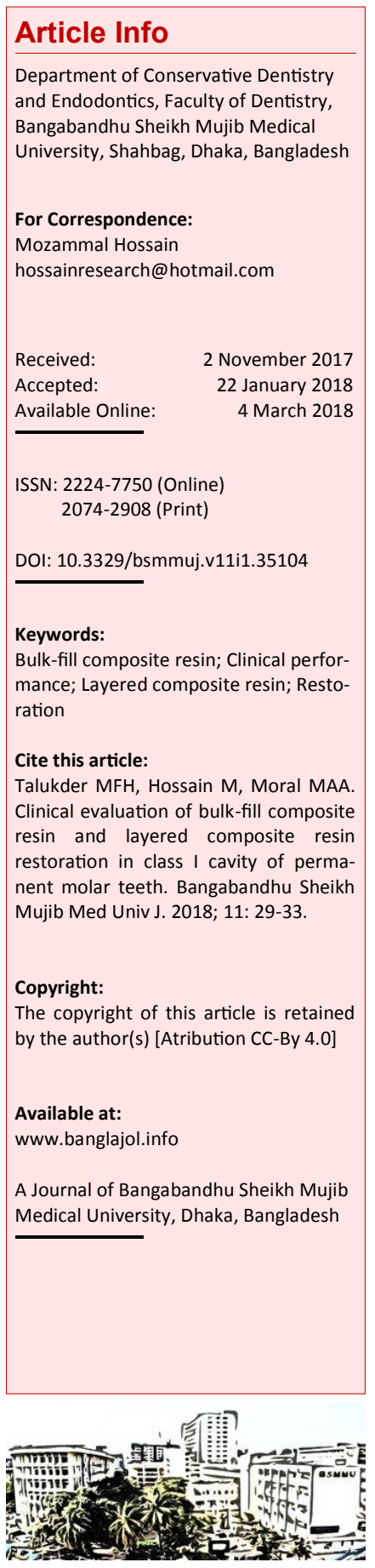

\section{Abstract}

The purpose of the present study was to compare the clinical performance of bulk-fill composite resin with that of layered composite resin restorations in occlusal class I cavity of permanent molar teeth. This study includes a total of 104 teeth having class I cavity of 3-4 mm depth in the occlusal surface. They were restored either with layered composite resin or bulk-fill composite resin of 52 teeth in each group. To observe the retention, marginal adaptation and color match, all restorations were assessed by using modified United States Public Health Services (USPHS) and Ryge's criteria at baseline, 3, 6 and 12 months. The results of 12 months observation showed that retention of 38 layered composite and 48 bulk-fill composite resin restorations revealed alpha rating, which was statistically significant. The marginal adaptation of 37 layered composite resin and 47 bulk-fill composite resin restorations also demonstrated alpha rating and the differences between two groups were statistically significant. However, there were no significant differences between the layered composite resin and bulk-fill composite resin restorations in respect to color match. It can be concluded that bulk-fill composite resin showed better clinical outcome than that of layered composite resin in the restorations of occlusal class I cavity.

\section{Introduction}

Composite resin is one of the most common non-metallic restorative materials which is used for tooth-colored, directly placed restorations. The material is based on a polymer resin matrix with filler particles. Most are based on the bisGMA resin developed by Bowen in 1962. Furthermore, the use of filler particles provide improved translucency, reduce the coefficient of thermal expansion and make the material more wear resistant. However, the incidence of secondary caries, marginal microleakage due to polymerization shrinkage and post-operative sensitivity still remains.

Polymerization shrinkage results in microleakage and debonding of the restoration. The clinical effects are increased risk of secondary caries and post-operative sensitivity. $\underline{1}$ Various approaches have been developed to solve some of the deficiencies of direct placement composites.? To reduce the rate of polymerization shrinkage, incremental filling techniques have been recommended which is believed to be effective in reducing polymerization shrinkage and minimize the total volumetric shrinkage. In this technique, the thickness of each layer is limited to $2 \mathrm{~mm}$ for optimal polymerization and degree of conversion. 3 Even though incremental layering technique ensure adequate polymeri- zation of the composite resin but air entrapment between the different layers may occur. Moreover, the incremental placement of composite resin requires longer period of time which increases the time required for restorative procedure. There is still controversy among the researchers and practitioners in respect to incremental layering versus bulk placement of composite resin. Therefore, the addition of small particles (e.g. nanoparticles) and placement of composite resin up to $4 \mathrm{~mm}$ is recommended to reduce polymerization shrinkage thus eliminating the need for technique sensitive layering protocol. 5

In recent years, bulk-fill composite has been developed to reduce the shrinkage stress during polymerization and offer much greater depth of cure. This is achieved by the addition of fillers such as barium aluminum silicate filler, ytterbium trifluoride and mixed oxides. Furthermore, a prepolymer filler (a shrinkage stress reliever) has been added with silanes, which is believed to reduce shrinkage stress. Furthermore, bulk placement can be possible up to $4 \mathrm{~mm}$ by $10 \mathrm{sec}$ curing time. 6 Therefore, it can be consider that bulk-fill composite resin is suitable to reduce polymerization shrinkage as well as post-operative sensitivity.

The aim of the present study was to compare 
the clinical performance of bulk-fill composite resin and layered composite resin restorations in permanent molar teeth at baseline, 3, 6 and 12 months interval by using the modified USPHS criteria.

\section{Materials and Methods}

This study includes a total of 104 teeth having class I cavity of 3-4 mm depth in the occlusal surface. After mouth preparation of each patient, isolation of tooth was done with cotton roll and saliva ejector. Class I cavities were prepared by using round burs in low-speed hand piece to remove carious tissue and then cleaned with normal saline.

\section{Restorations}

In Group I (layered composite resin), following completing cavity preparation, all the cavities were subjected to the one step self-etch adhesive system (3M ESPE's AdperTM Easy Self-Etch Adhesive) according to manufacturer's instructions. Adhesive systems were then cured for $10 \mathrm{sec}$ with light cure machine. The restoration was placed with layered composite resin (3M ESPE's Filtek P60) with increments (Figure 1); each layer not being more than $2 \mathrm{~mm}$ and was cured for $20 \mathrm{sec}$. Finishing of the restoration was carried out by adapting occlusion and articulation using fine-grit diamonds. Finally, the composite restoration was polished using super snaps polishing kit.

In Group II (bulk-fill composite resin), after completing cavity preparation, all the cavities were assigned to the one step self etch adhesive system (Tetric N-Bond Self-Etch) according to manufacturer's instructions. Adhesive systems were then cured for $10 \mathrm{sec}$ with light cure machine. The bulkfill composite resin (Ivoclar Vivadent's Tetric NCream Bulk-fill) was placed into the cavity by single layer and polymerized for $10 \mathrm{sec}$ (Figure 2). Finishing of the restoration was carried out by adapting occlusion and articulation using fine-grit diamonds. Finally, the composite restoration was polished using super snaps polishing kit.

\section{Follow-up visit}

The patients were recalled at 3, 6, 12 months and clinical assessment were performed as follows: Each restoration was evaluated with dental mirror and probe by two experienced clinician who were not involved with the study. Restoration was rating at

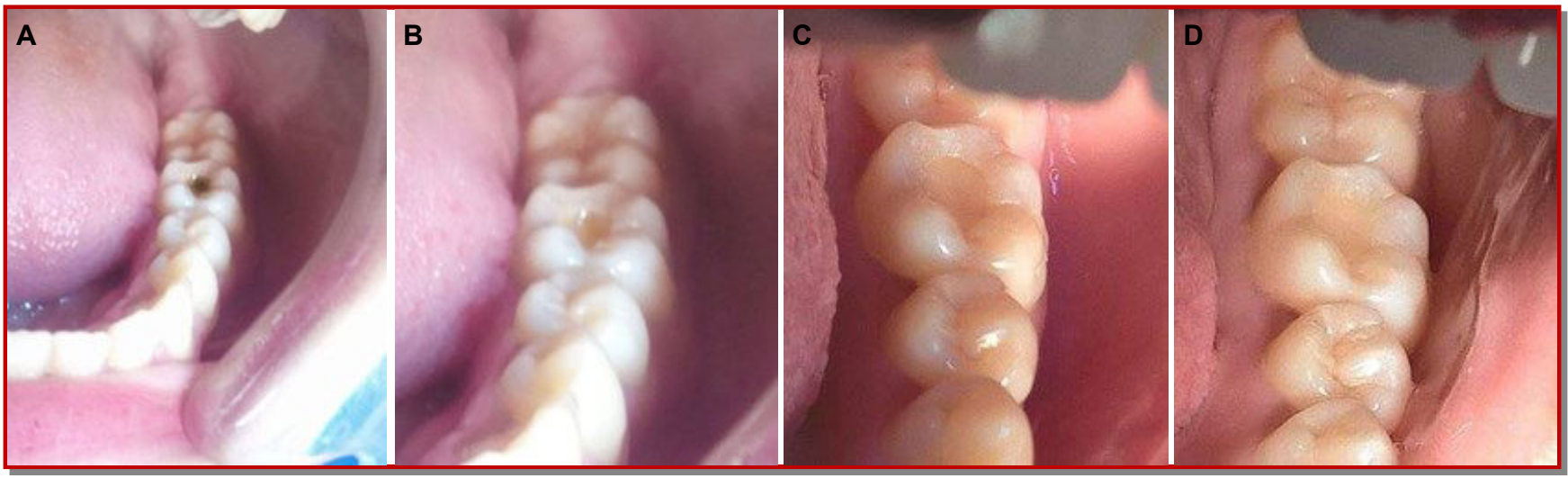

Figure 1: Representative photographs of a restoration with layered composite resin (A: Pre-operative; B: Following cavity preparation; C: Restored with layered composite resin; D: At 12 months)

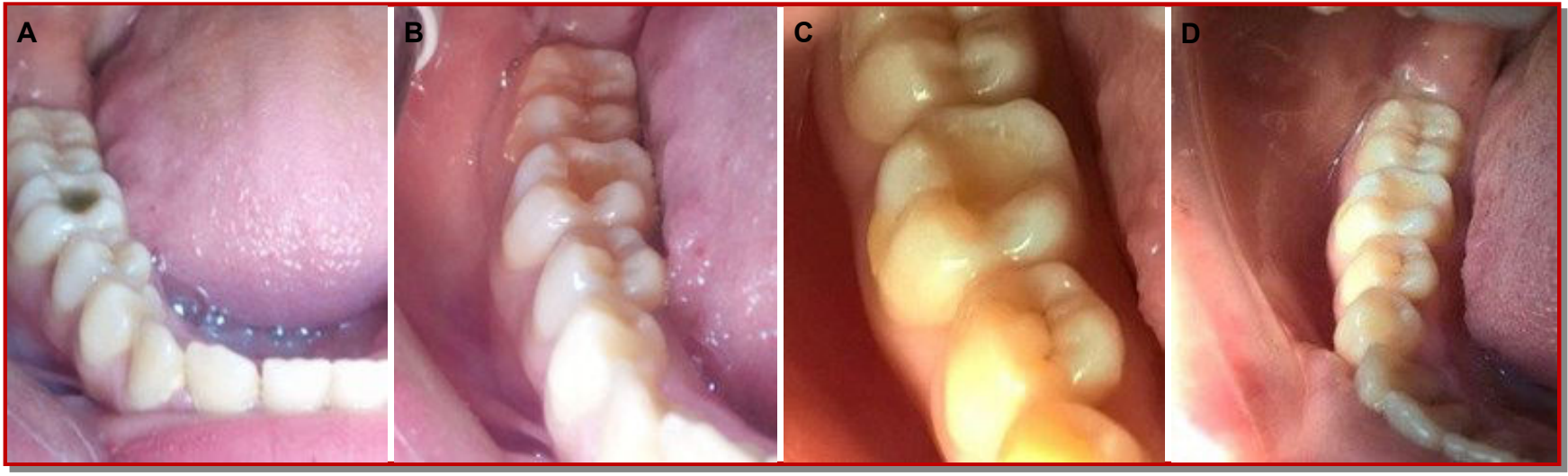

Figure 2: Representative photographs of a restoration with bulk-fill composite resin (A: Pre-operative; B: Following cavity preparation; C: Restored with bulk-fil composite; D: At 12 months) 
baseline evaluation, 3, 6 and 12 months by using modified United States Public Health Services (USPHS) and Ryge's criteria (Figure 1, Figure 2). This clinical assessment method resulted in ordinal structured data for the outcome variable.

\section{Results}

The results of 38 layered composite resin and 48 bulk-fill composite resin restorations revealed alpha rating (restoration were fully intact into the cavity) at the end of one year, which was statistically significant $(p<0.05)($ Table I). Furthermore, the results of marginal adaptation of 37 layered composite resin and 47 bulk-fill composite resin restoration demonstrated alpha rating (there were no visual evidence of crevice along the margin and explorer did not catch) at the end of one year and the differences between two groups were statistically significant $(p<0.05)$. Regarding color match, 40 layered composite resin and 45 bulk-fill composite resin restorations showed alpha rating (matched with the respective tooth) at the end of one year. Furthermore, there were no significant differences between layered composite resin and bulk-fill composite resin restorations in respect to color match were found.

\section{Discussion}

The results of the present in vivo study showed that direct restoration by 3-4 $\mathrm{mm}$ bulk-fill composite resin showed better clinical outcome than that of

\begin{tabular}{|c|c|c|c|c|}
\hline \multicolumn{5}{|c|}{ Table I } \\
\hline \multicolumn{5}{|c|}{ Results of clinical assessment at 12 months ( $n=104$ teeth) } \\
\hline & & Group I & Group II & \\
\hline Evaluation & Score & $(n=52)$ & $(n=52)$ & $\mathrm{p}$ value \\
\hline \multirow[t]{4}{*}{ Retention } & Alpha & 38 & 48 & \\
\hline & Bravo & 10 & 2 & 0.011 \\
\hline & Charlie & 0 & 0 & \\
\hline & Missing cases & 4 & 2 & \\
\hline \multirow[t]{4}{*}{ Marginal adaptation } & Alpha & 37 & 47 & \\
\hline & Bravo & 3 & 2 & 0.033 \\
\hline & Charlie & 8 & 1 & \\
\hline & Missing cases & 4 & 2 & \\
\hline \multirow[t]{4}{*}{ Color Match } & Alpha & 40 & 45 & \\
\hline & Bravo & 5 & 3 & 0.621 \\
\hline & Charlie & 3 & 2 & \\
\hline & Missing cases & 4 & 2 & \\
\hline
\end{tabular}

layered composite resin restorations. When these restorations were examined at 3, 6 and 12 months, it was found that most of the bulk-fill restorations showed acceptable color matching, marginal adaptation as well as retention. Furthermore, the clinical outcome of bulk-fill composite resin restorations were also statistically significant than that of layered composite resin restorations at 6 and 12 months observation period. The overall success rate in the retention of bulk-fill in the present study was $96 \%$ which was almost similar to a study. $?$

Several in vivo and in vitro studies have been performed to compare the efficacy of bulk-fill composite resin with that of layered composite resin.

The results of the present study had similarities and dissimilarities with that of previous studies. Kapoor et al. (2016) $\underline{8}$ indicated that bulk-fill composites revealed better adaptability and less gap formation at the pulpal floor than the incremental composites. Furthermore, Roggendorf et al. (2015)9 revealed that when direct resin composite was placed as $4 \mathrm{~mm}$ bulk-fill dentin replacement, good performance was achieved in the study. On the other hand, Heintze et al. (2015) 10 reported that the marginal integrity of composite resin placed in one increment was similar when compare to restorations placed in several increments. Furthermore, the differences between the resin restorations placed in bulk and those placed in several increments was not statistically significant.

However, at 6 month observation period, 2 bulk-fill composite resin restorations showed Charlie rating (completely missing), which was replaced by a new restoration and therefore dropped from the study. Furthermore, at 12 months observation period, another 2 restorations were partially lost (Bravo rating) which was not replaced but repaired by bulk -fill composite resin. None of the restoration was lost at this observation period. On the other hand, 6 layered composite resin restorations showed Bravo and 4 revealed Charlie rating at 6 months followed by 10 Bravo at 12 months. The reasons of loss of retention in both bulk-fill and layered composite restorations were not clarified in the present study. But it may be due to loss of adhesion either by polymerization shrinkage or due to degradation of the adhesive itself. Other possible reasons of partial or complete loss of retention might be due to fracture or crack formation, inadequate elastic modulus and the flexibility of restorative material.

Furthermore, the choice of adhesive system becomes important especially in large cavities when stress is higher as better retention could be achieved when restorative material is used along with bonding system.11 Furthermore, Peumans et al. (2005) 12 indicated that adhesives provide good clinical performance. Therefore, based on the present study and together with the previous 
studies it can be considered that loss of retention occur due to multi factorial etiology such as patient selection, location, occlusal stress, presence of sclerotic dentin, shape of the lesions, as well as on the properties of the materials used. 13

Regarding marginal adaptation, although bulk-fill composite resin restorations showed significantly better adaptation than that of layered composite resin restorations, but at 6 months 1 restorations showed Bravo at 6 months followed by 2 Bravo and 1 Charlie rating at 12 months. Loss of marginal adaptation of these cases was due to chipping at margin of the restoration and they were not replaced but repaired with same material. A careful examination of these restorations revealed that all chipping occurred due to direct contact with opposing cusp. Direct composite restorations should not be placed in direct contact with opposing cusp. This is also supported by a previous study. $\underline{14}$

When the color of the restorations was verified, it was found that 3 bulk-fill composite resin restorations were slightly mismatched with the same tooth at 6 months followed by 3 Bravo and 2 Charlie rating at 12 months. On the other hand, 5 of layered composite resin restorations showed Bravo and 3 revealed Charlie rating at 12 months. Careful examinations of these restorations showed that mismatch of the color of the restorations were due to body discoloration by gradual discoloration of the monomer component of the material. The results found in the present study were also correspondent to the previous study. $\underline{15}$ Furthermore, no restorations showed marginal discolorations which indicates that neither secondary caries nor any postoperative sensitivity could occur at this stage. The results between two groups in respect to incidence of color change, they were not statistically significant ( $\mathrm{p}>0.05)$.

In this study, bulk-fill composite resin demonstrated significantly better results than that of layered composite resin. There are several reasons of better results achieved by bulk-fill composite resin than that of layered composite resin restoration. It can minimizes polymerization shrinkage and increases the wear resistance due to addition of fillers such as barium aluminum silicate filler, ytterbium trifluoride and mixed oxides. Furthermore, the filler used in this composite resin associated with silanes acts as a unique shrinkage stress reliever. Moreover, the addition of ivocerin (dibenzoyl germanium derivatives) with the initiator helps in the curing of posterior restorations in large increments up to 4 $\mathrm{mm}$ without hampering its retention and marginal adaptation as well as translucency or color.16 On the other hand, increment layered technique for restorative material condensation is considered helpful for reducing polymerization shrinkage, but previous study have indicated that incrementally placed composite resin are associated with voids or moisture contamination between each layer of composite resin that may cause bond failures between increments. 4 The difficulty in placement of composite resin due to limited access in conservative preparations and the increased time required to place and polymerize each layer is responsible for voids or moisture contamination. .4 Compared to the incremental build-up technique of the restoration, bulkfill composites require less chair side time and are more predictable, making the restorative process comfortable for the patient. 6 Furthermore, bulk-fill composite resin had good adaptation, elasticity and low polymerization shringkage stress which may reduces microleakage, postoperative sensitivity and secondary caries due to improved depth of cure (at least $4 \mathrm{~mm}$ ) and eliminates the steps of layering. $\underline{17,18}$ As this material can be cured in bulk which may allow the curing light to sufficiently penetrate to the bottom of single increment layer.17,18 The use of bulk-fill composite resin restoration in posterior teeth of the present study was limited to class I cavities, it can be said that polymerization stresses could be minimum in these restorations. This might be the possible reasons of better clinical results with bulk-fill composite resin of the present study.

\section{Conclusion}

Bulk-fill composite resin is superior to layered composite resin in respect to retention and marginal adaptation in class I restorations of permanent molar teeth.

\section{Ethical Issue}

The research protocol was approved by the committee and permission for the study was taken from the Institutional Review Board of Bangabandhu Sheikh Mujib Medical University.

\section{Acknowledgement}

This work was supported by the Research Grant for Student, Bangabandhu Sheikh Mujib Medical University. 


\section{References}

1. Loguercio AD, Bauer JR, Reis A, Grande RH. Microleakage of packable composite in class II restorations. Quintessence Int. 2004; 35: 29-34.

2. Carvalho RM, Pereira JC, Yoshiyama M, Pashley $\mathrm{DH}$. A review of polymerization contraction: The influence of stress development versus stress relief. Oper Dent. 1996; 21: 17-24.

3. Leprince JG, Palin W, Julie Vanacker J, Sabbagh J, Devaux J, Leloup G. Physico-mechanical characteristics of commercially available bulk fill composites. J Dent. 2014; 2: 21-25

4. El-Safty S, Silikas N, Watts DC. Creep deformation of restorative resin-composites intended for bulk-fill placement. Oper Dent. 2012; 40: 622-35

5. Mackenzie L, Shortall AC, Burke FJ. Direct posterior composites: A practical guide. Dent Update. 2009; 36: 71-72.

6. Giachetti L, Scaminaci RD, Bambi C, Grandini R. A review of polymerization shrinkage stress: Current techniques for posterior direct resin restorations. J Contem Dent Prac. 2006; 7: 79-88.

7. Frankenberger R, Schulz M, Roggendorf MJ. Bulkfill vs. layered resin composites: Six month report. J Dent. 2013; 38: 216-25.

8. Kapoor N, Bahuguna N, Anand S. Influence of composite insertion technique on gap formation. Br Dent J. 2016; 10: 337-44.

9. Roggendorf MJ, Krämer N, Appelt A, Naumann $\mathrm{M}$, Frankenberger R. Marginal quality of flowable $4 \mathrm{~mm}$ base vs. conventionally layered resin composite. J Ad Dent. 2015; 17: 147-54.
10. Heintze SD, Monreal D, Peschke A. Marginal quality of class II composite restorations placed in bulk compared to an incremental technique: Evaluation with SEM and stereomicroscope. J Ad Dent. 2015; 17: 147-54.

11. Xu HH. Dental composite resins containing silicafused ceramic single-crystalline whiskers with various filler levels. J Dent Res. 1999; 78: 1304-11.

12. Peumans $\mathrm{M}$, Kanumilli $\mathrm{P}$, DeMunck J, VanLanduyt K, Lambrechts P, VanMeerbeek B. Clinical effectiveness of contemporary adhesives: A systematic review of current clinical trials. Dent Mater. 2005; 21: 864-81.

13. Pecie R, Krejci, Garcia-Godoy F, Bortolotto T. Noncarious cervical lesions (NCCL): A clinical concept based on the literature review. Part 2 restoration. Am J Dent. 2011; 24: 183-92.

14. Hossain M, Kawakami S, Shimokobe H. Effect of gap dimension on wear of prototype glass inomer luting cements in margin of ceramic restorations: In vivo. J Conserv Dent. 1995; 38: 212-24.

15. Moszner N, Fischer U, Ganster B, Liska R, Rheinberger $\mathrm{V}$. Benzoyl germanium derivatives as novel visible light photo initiators for dental materials. Dent Mater. 2008; 24: 901-07.

16. Sesemann MR. Placing a bulk fill composite to achieve predictable and esthetic posterior restorations. Oral Health J. 2012; 102; 43-48.

17. Ahmad I. Deep resins, white fillings: A new technique for composite restorations. J Cosmet Dent. 2013; 3: 22-31.

18. Ryge G, Snyder M. Evaluating the clinical quality of restoration. J Am Dent Assos. 1973; 87: 369-77. 